\title{
Étude de la productivité des parcours de la zone d'Ain-Leuh (Moyen Atlas, plateau Central). II. Modélisation de la production d'herbe : interactions climats $\mathrm{x}$ sol $\mathrm{x}$ recouvrement arboré

\author{
M Qarro 1, FX de Montard 2 \\ ${ }^{1}$ ENFI BP 511 Tabriquet, Salé, Maroc; \\ 2 INRA agronomie, 12 avenue du Brézet, 63039 Clermont-Ferrand, France
}

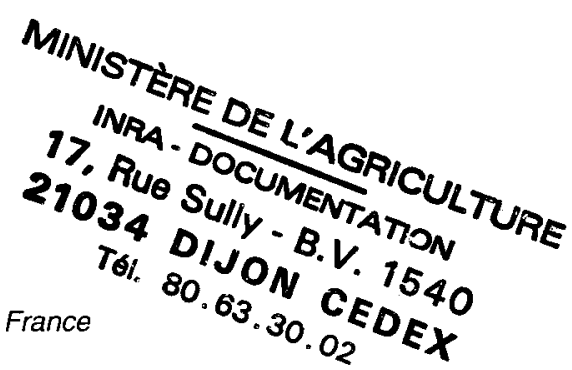

(Reçu le 3 juin 1991; accepté le 2 juin 1992)

\begin{abstract}
Résumè - La production d'herbe a été mesurée en 1983 et 1984 dans les parcours boisés du Moyen Atlas et du plateau Central dans la région d'Aïn Leuh sur 4 sites représentatifs des ensembles bioclimatiques semi-aride (chênaie verte à oxycèdre), subhumide (chênaie verte pure du Dir), et humide (cédraie mixte à chêne vert et pelouse asylvatique); les mesures ont été effectuées dans des enclos protégés du pâturage en 5 coupes échelonnées de fin février à mi-juillet sur un réseau de placettes représentatives d'un gradient de recouvrement arboré (0-90\%). La récolte d'hiver, en fin février, est principalement expliquée par la hauteur des précipitations de septembre et octobre en interaction avec le taux d'azote de l'horizon supérieur du sol dans le cas des sols squelettiques du semi-aride, ou avec le taux de sable grossier pour les sols argileux des ensembles subhumide et humide (taux de pénétration des pluies). Les 4 récoltes échelonnées de mars à juillet sont expliquées par l'interaction de la ressource énergétique globale et d'une fonction du couvert arboré $R$, dissymétrique à maximum (100-R) $e^{R / B}$, ( $B$ variant entre 50 et 90 selon le bioclimat et le type de plante herbacée, fixatrice d'azote ou non). L'humidité du climat intervient additivement en fonction du niveau d'alimentation en eau en climats subhumide et humide et interactivement en fonction du taux de jours de pluie en climat semi-aride.
\end{abstract}

agriculture sylvo-pastorale / parcours / production / matière sèche / facteur climatique / couvert arboré

Summary - A study of dry matter production in rough pastures of Ain-Leuh (Middle Atlas, central plateau). II. Model of herbage production: climate-soil-tree cover interactions. Herbaceous production was measured in 1983 and 1984 in the Middle Atlas and central plateau rangelands and grazed forests, in the Ain-Leuh region, at 4 characteristic sites in 4 bioclimatic zones: semi-arid (Quercus ilex and Juniperus oxycedrus forest), sub-humid (pure Quercus ilex forest), and humid (Cedrus atlanticus and Quercus ilex forest, unsylvatic low grass at the highest site); measurements were made in areas protected from sheep and goat grazing, through 5 successive cuts from late February to mid-July, on plots varying in tree cover from 0 to $90 \%$. Late February yield was mainly explained through the degree of precipitation occurring in both September plus October, interacting with the levels of soil nitrogen in poor soils of the Quercus and Juniperus zone, or level of rough sand in clay soils of the 3 upper zones. Yields from 4 successive cuts from the end of March to mid-July were mainly explained through interactions between radiative energy and a function of tree cover rate $(100-R) e^{R / B}$; the $B$ value was 50 in the semi-arid zone, and 90 for non-legume and 50 for legume plants in the sub-humid/humid zone). A climate humidity factor, the ETR/ETP ration in sub-humid and humid zone or the frequency of rainy days in the semi-arid zone, had an additive and an interactive effect respectively.

silvopastoral agriculture / range / production / dry matter / nitrogen output / climatic factor / tree cover rate 


\section{INTRODUCTION}

Les parcours de la région d'Aïn-Leuh couvrent 4 ensembles bioclimatiques, la chênaie verte à oxycèdre sur le plateau Central en climat semi-aride (900-1300 m d'altitude), la chênaie verte pure du Dir en climat subhumide (1300-1700 m), la cédraie mélangée de chêne vert en climat humide frais et les pelouses asylvatiques des dépressions gélives en climat humide froid dans le Moyen Atlas $(1700-2200 \mathrm{~m})$. Le recouvrement arboré est extrêmement variable, et dépend de la densité d'occupation humaine et de l'intensité des pratiques agricoles et pastorales qui ont conduit dans certains secteurs à la disparition de la forêt.

Précédemment (Qarro, 1985; Qarro et de Montard, 1989) ont été présentés les types de sol, les variations climatiques saisonnières, les relevés floristiques et les résultats de productivité du tapis herbacé au cours des campagnes 1982-1983 et 1983-1984, en fonction du rythme de coupe et du taux de couvert arboré, dans 4 stations caractéristiques de ces bioclimats.

Les principales conclusions étaient l'intérêt de l'exploitation fréquente ( 4 coupes de mars à juillet sont légèrement plus productives au total que 3 ou 5 coupes, mais nettement plus productives qu' 1 ou 2 coupes) et la mise en évidence d'une compatibilité entre la production d'herbe et le maintien d'un couvert arboré jusqu'à un taux de $60-70 \%$ (un couvert arboré de $30-60 \%$ a entraîné une diminution des récoltes d'environ $30 \%$ en 1984, année humide, mais n'a pas eu d'effet significatif en 1983, année à déficit hydrique précoce). La présente étude va au-delà d'une analyse de la production annuelle pour passer au niveau d'une analyse des facteurs de production à l'échelle mensuelle.

\section{Objectif de l'étude}

L'objectif de la présente étude est d'effectuer, sur les données de production mensuelles rassemblées par M Qarro (1985), un essai de modélisation de la production d'herbe prenant en compte les interactions climat $x$ sol $x$ recouvrement arboré; il s'agit d'intégrer les rósultats de production de MS obtenus dans un modèle agronomique cohérent pour un ou plusieurs ensembles bioclimatiques et d'interpréter la hiérarchie des variables observée en rapport avec les types de climat et de végétation. L'examen de la littérature permet de se rendre compte de la di- versité des réponses observées selon les milieux. Cette étude tentera de préciser le rôle du recouvrement arboré au cours des saisons dans les parcours du Moyen Atlas.

\section{Les effets du couvert arboré sur la strate herbacée : état des connaissances}

Les effets de la présence d'une strate arborée sur la production de la strate herbacée sous-jacente ont été étudiés sous des climats divers, soit en végétation naturelle, soit dans des plantations résineuses; les résultats sont parfois contradictoires :

Dans les parcours, la présence d'arbres isolés se traduit tantôt par une baisse de la production d'herbe à l'abri de la couronne (Juniperus virginiana; Engle et al, 1987) par rapport à la pleine lumière tantôt par une augmentation faible (Pinus sabiniana) à très importante au moment du pic de production (Quercus douglasii et Quercus wislinezir; Frost et Mc Dougald, 1989). Sous Acacia caven, Ovalle et Avendano (1987) observent que la production de matière sèche diminue en période humide avec des couverts arborés croissants, et, au contraire augmente fortement avec le couvert arboré à la période d'établissement de la sécheresse estivale; le bilan sur l'année a été un effet positif jusqu'à un recouvrement arboré de $80 \%$.

Dans les forêts de pins cultivées de Caroline du Sud, Leithead (1970, cité par Richard, 1987), a établi une courbe de production d'herbe en fonction de l'ombrage, qui commence par un plateau jusque vers $10-15 \%$ d'ombrage, et s'incline ensuite selon une pente descendante assez régulière; la production devient nulle au-delà de $70 \%$ d'ombrage. D'autres auteurs (Dodd et al, 1972; Clary, 1975; cités par Richard, 1987) ont proposé un modèle d'ajustement de la production d'herbe aux paramètres de la géométrie du couvert; Lewis (1989) établit une relation directe avec l'âge de la plantation, Woods et al (1982) avec la surface terrière; pour Knowles (1989) le meilleur paramètre pour exprimer la production herbacée sous Pinus radiata est la longueur de la couronne. Ces formules empiriques sont pratiques dans un contexte donné, mais non explicatives ni généralisables.

En réalité, le sens de la réponse au couvert arboré est très variable et dépend de la nature du couvert. Lorsqu'on entre dans l'analyse du fonctionnement du système écologique, la compétition pour la lumière n'est pas le seul facteur en jeu : 
a) L'accumulation de litière peut constituer un facteur limitant la production d'herbe, soit par occupation du sol, soit par blocage du cycle de l'azote dans le cas d'un rapport C.N-1 élevé (Gaines et al, 1954) : la composition des litières, leur vitesse de recyclage et la quantité d'éléments nutritifs libérés conditionnent fortement le fonctionnement et la productivité de la strate herbacée; elles dépendent étroitement de la nature de l'essence dominante (Zimka et Stachurski, 1975, 1976; Eason, 1991).

b) Le décalage phénologique entre l'arbre et l'herbe peut être remarquable; c'est le cas d'Acacia caven étudié par Ovalle et Avendano (1987) sous climat méditerranéen au Chili, arbre dont les feuilles s'étalent vers la fin de la période de végétation des graminées, lorsque la sécheresse est installée; en général, les caducifoliés sont plus ou moins favorables selon la tardivité de leur débourrement : les espèces marcescentes, les résineux clairs (pins, cèdres), les espèces sempervirentes, les résineux sombres constituent, dans l'ordre cité, des espèces de moins en moins favorables à la réception de la lumière par la strate herbacée.

c) Ovalle et Avendano (1987) observent une dominance des composées de valeur médiocre (Hypochaeris radicata) à découvert, de Lolium multiflorum sous couvert d'Acacia caven, et une phénologie retardée de $7-16$ j selon les espèces sous couvert.

d) Bien que l'interception d'une partie des précipitations par les couronnes constitue un handicap, le couvert arboré diminue aussi le rayonnement intercepté et la demande évaporative; il en résulte, sous climat méditerranéen, une augmentation de l'humidité du sol et une prolongation de la période de pousse herbacée active au-delà de la date de cessation des pluies (Joffre, 1987; Étienne et Msika, 1987; Hubert, 1989). Dans le cas d'Acacia caven (Ovalle et Avendano, 1988), la transmission de la lumière a été de 30 à $50 \%$ et il en est résulté une augmentation de l'humidité disponible dans le sol sur 3 mois et un retardement de plus de $20 \mathrm{j}$ de son épuisement, en début d'été.

e) Dans la Dehesa (Vacher et al, 1985),

- forêt claire de Quercus rotundifolia dans laquelle les couronnes des arbres couvrent environ $25 \%$ de l'espace (30-50 arbres/ha couvrant chacun 30-50 $\mathrm{m}^{2}$ )

- Joffre (1987) explique ces phénomènes par une nette amélioration de la pénétration des précipitations arrivant au sol et de la capacité de rétention de l'eau sous couvert par rapport aux zones à découvert. II en résulte une évapo- transpiration réelle (ETR) plus faible de l'herbe abritée, mais une ETR supérieure de 150-200 $\mathrm{mm}$ pour le système total «arbre + herbe» par rapport à l'herbe située à découvert.

f) Dans une plantation de pin maritime des Landes, Berbigier et al (1991) ont montré que la transpiration de la strate herbacée constituée de molinie, représente $45-54 \%$ de la transpiration totale du couvert; pendant la journée, la transpiration du houppier exprimée en proportion de l'énergie de vaporisation incidente à son niveau (exprimée en $\mathrm{mm}$ ) diminue régulièrement, de 55 à $15 \%$, de mai à septembre, tandis que la transpiration de la molinie ne descend pas en dessous de $60 \%$ de l'énergie incidente au-dessus du sous-bois; celle-ci est en moyenne de $30 \%$ de l'énergie incidente au niveau des houppiers. La sévérité du stress hydrique apparaît donc beaucoup moins grande en sous-bois qu'au niveau du houppier. Nous verrons que ce type de relation peut expliquer certains de nos résultats.

\section{MATÉRIELS COLLECTÉS ET MÉTHODES}

\section{Choix des stations et placettes de mesures}

Les parcours étudiés s'étendent sur le territoire de la commune rurale d'Aïn-Leuh. La production de matière sèche de la strate herbacée a été mesurée dans une station de chaque bioclimat le long d'un gradient de recouvrement arboré croissant; ces mesures ont concerné 9 sous-stations dans la chênaie verte à oxycèdre vers $1050 \mathrm{~m}$ d'altitude (recouvrement : 0$69 \%$ ), 8 sous-stations dans la chênaie verte pure du Dir vers $1600 \mathrm{~m}$ (recouvrement : 0-91\%), 6 sousstations dans la cédraie vers $1800 \mathrm{~m}$ (recouvrement : $0-83 \%$ ) et une sous-station dans la pelouse de cuvette située vers $2000 \mathrm{~m}$ et dépourvue d'arbres. L'ensemble concerne donc 24 sous-stations réparties dans 4 stations caractéristiques des 4 bioclimats.

\section{Modalités de récolte et observations sur le milieu}

Les récoltes ont été effectuées en fin d'hiver, puis mensuellement jusqu'au 20 juillet. Les observations météorologiques ont porté dans chaque station sur les précipitations, les températures mini-maxi journalières sous abri placé à découvert, les phénomènes de rosée, brouillard, gelée et ennuagement; l'ETP a été évaluée selon la méthode proposée par Thornwaite. Les bilans hydriques ont été calculés mensuellement ou par période entre 2 coupes sur la base 
d'une réserve utile de $100 \mathrm{~mm}$; cette hypothèse moyenne englobe l'ensemble des taux de couvert; son adoption vise à faire apparaître a posteriori les effets correctifs du recouvrement et de la profondeur de sol, sans hypothèse préalable.

Dans chacune des 24 sous-stations choisies : le site, le sol et la végétation ont été étudiés ainsi : la pente et l'exposition ont été déterminées; un densiomètre à miroir carré comportant 25 carreaux a permis d'évaluer le taux de couvert arboré. Les profils de sol ont été décrits et caractérisés et les analyses ont porté sur granulométrie, $\mathrm{pH}, \mathrm{C}, \mathrm{N}, \mathrm{P}$ assimilable, cations échangeables, capacité d'échange et humidité à pF 2,5 et $\mathrm{pF} 4,2$ pour les différents horizons (1-3 selon le profil). La composition floristique a été déterminée par la méthode du relevé phytosociologique et les productions de matière sèche ont été évaluées par coupe sur des placettes de $1 \mathrm{~m}^{2}$ randomisées dans un rectangle de $2 \mathrm{~m}$ sur $10 \mathrm{~m}$ orienté perpendiculairement à la pente. Selon les placettes, la végétation a été coupée de 1-5 fois entre le début mars et la mi-juillet.

La présente étude concerne seulement le cas de 5 coupes (2 placettes par sous-station) réalisées à un rythme mensuel; ce rythme est celui qui simule le mieux le passage fréquent des ovins en parcours. Les conditions régnant pendant la repousse prévalent sur d'éventuels arrière-effets d'une coupe sur la suivante, l'effet de la coupe qui rabat la végétation au ras du sol étant très prépondérant; les récoltes successives sont donc considérées comme des résultats indépendants.

Les principales caractéristiques physiques et chimiques de l'horizon supérieur des sols, la profondeur du sol, le taux de recouvrement arboré et la contribution des légumineuses herbacées des 24 sous-ștations sont

Tableau I. Principales caractéristiques des sous-stations.

$\begin{array}{lllllllllllll}\text { SSt } & R & L g & \operatorname{Pr} & \mathrm{Ar} & \mathrm{Sg} & \mathrm{N} & \mathrm{C} / \mathrm{N} & \mathrm{K} & \mathrm{Ca} & \mathrm{Mg}\end{array}$

Chênaie à oxycèdre

$\begin{array}{ll}1 & \\ 2 & 3 \\ 3 & 19 \\ 4 & \\ 5 & \\ 6 & 6 \\ 7 & 13 \\ 8 & 70 \\ 9 & \end{array}$

0
33
19
0
0
62
13
70
0

$\begin{array}{rr}0 & 37 \\ 3 & 34 \\ 13 & 29 \\ 1 & 30 \\ 11 & 30 \\ 2 & 38 \\ 10 & 30 \\ 3 & 25 \\ 12 & 30\end{array}$

$\begin{array}{ll}37 & \\ 34 & 13 \\ 29 & 26 \\ 30 & 27 \\ 30 & 17 \\ 38 & 18 \\ 30 & 24 \\ 25 & 18 \\ 30 & 13\end{array}$

1
13
26
27
17
18
24
18
13

75
46
29
33
39
28
29
34
38

1,5
1,3
1,7
1,7
2,2
2,3
2,2
1,6
1,4

9,4
5,7
7,9
7,8
5,0
7,1
6,2
7,7
7,1

0,32
0,25
0,25
0,19
0,12
0,12
0,25
0,12
0,19

$\begin{array}{rr}5,0 & 1,5 \\ 5,8 & 1,9 \\ 7,5 & 3,0 \\ 6,8 & 1,7 \\ 9,0 & 2,0 \\ 7,8 & 2,0 \\ 9,8 & 1,7 \\ 7,5 & 2,2 \\ 10,0 & 1,5\end{array}$

\section{Chênaie verte pure}

$\begin{array}{rrr}10 & 62 & 47 \\ 11 & 38 & 75 \\ 12 & 0 & 28 \\ 13 & 0 & 12 \\ 14 & 19 & 46 \\ 15 & 91 & \\ 16 & 20 & 11 \\ 17 & 39 & 62\end{array}$

$\begin{array}{rl}47 & 30 \\ 75 & 50 \\ 28 & 40 \\ 12 & 38 \\ 46 & 33 \\ 0 & 50 \\ 11 & 50 \\ 62 & 40\end{array}$

48
53
53
60
44
19
21
40

2
2
1
1
2
3
5
3

$\begin{array}{rr}3,5 & 9,5 \\ 3,6 & 10,1 \\ 5,0 & 9,1 \\ 5,1 & 9,2 \\ 6,8 & 1,6 \\ 13,4 & 14,8 \\ 3,1 & 9,8 \\ 5,2 & 11,3\end{array}$

$\begin{array}{lll}1,15 & 25,6 & 11,7 \\ 1,30 & 25,0 & 11,1 \\ 1,34 & 27,5 & 11,4 \\ 0,96 & 27,5 & 12,9 \\ 1,66 & 34,3 & 12,7 \\ 1,15 & 50,0 & 16,3 \\ 0,44 & 23,2 & 10,2 \\ 1,02 & 30,6 & 11,1\end{array}$

Cédraie et chêne vert

\begin{tabular}{|c|c|c|c|c|c|c|c|c|c|c|}
\hline 18 & 70 & 6 & 30 & 59 & 3 & 1,6 & 10,7 & 0,64 & 15,8 & 11,2 \\
\hline 19 & 75 & 6 & 75 & 28 & 18 & 5,0 & 9,0 & 1,02 & 17,5 & 4,1 \\
\hline 20 & 65 & 1 & 55 & 25 & 11 & 10,4 & 15,6 & 1,41 & 27,7 & 7,3 \\
\hline 21 & 0 & 15 & 30 & 36 & 4 & 3,7 & 9,0 & 0,83 & 18,8 & 11,0 \\
\hline 22 & 83 & 1 & 55 & 25 & 7 & 1,8 & 9,2 & 0,57 & 10,8 & 5,8 \\
\hline 23 & 0 & 2 & 60 & 38 & 5 & 4,3 & 11,1 & 1,21 & 17,5 & 7,5 \\
\hline \multicolumn{11}{|c|}{ Pelouse asylvatique } \\
\hline 24 & 0 & 16 & 55 & 28 & 17 & 5,2 & 10,2 & 2,31 & 19,3 & 9,7 \\
\hline
\end{tabular}

$S S t: n^{\circ}$ de la sous-station; $R$ : recouvrement arboré \%; $L g$ : somme des abondance-dominance des légumineuses $x$ 10; $P r$ : profondeur du sol en $\mathrm{cm} ; \mathrm{Ar}, \mathrm{Sg}$ : taux d'argile et de sable grossier en \%; $\mathrm{N}, \mathrm{Ca}, \mathrm{Mg}$ : teneurs en azote \% et en cations échangeables meq/100 g de l'horizon supérieur. 
données au tableau I. La nature et les profils de sols ont été décrits précédemment (Qarro, 1985); les sols de la station semi-aride sont des sols squelettiques sur shistes, argilo-sablo-limoneux; les sols des 3 autres stations sont argileux ou argilo-limoneux, sur calcaire pour la chênaie verte pure, sur calcaire dolomitique pour la cédraie et sur basalte pour la pelouse asylvatique.

\section{Nature des variables explicatives de la productivité}

La méthode envisagée pour construire un modèle représentatif de la productivité de l'herbe à partir des observations et analyses précédentes est la régression linéaire multiple pas à pas ascendante avec sélection progressive. Elle a été appliquée en utilisant un nombre limité de variables pertinentes du point de vue de la croissance de l'herbe : l'énergie globale incidente, le niveau d'alimentation en eau, la répartition des pluies (taux de jours de pluie) et des rosées, le taux de recouvrement arboré, la contribution des légumineuses et quelques caractéristiques importantes des sols (tableaux I et II). À défaut de mesure sur place du rayonnement photosynthétiquement actif intercepté au niveau de la strate herbacée $\left(P A R_{h}\right)$, celui-ci a été représenté indirectement par le produit du rayonnement absolu et du taux d'ouverture $R g(100-R)$; on a admis l'hypothèse de la linéarité à l'échelle du mois de la relation entre le produit $R g(100-R)$ et le $P A R_{h}$, hypothèse acceptable dans la mesure où les variations de $R g$ de mars à juillet sont très importantes.

L'objectif visé par cette analyse en régression multiple est d'identifier parmi les variables agronomiques ou écologiques les variables ou fonctions de variables les plus explicatives, d'établir une hiérarchie entre ces variables, et de déterminer leurs combinaisons, produit ou quotient, pouvant exprimer d'éventuelles interactions. Les variables des tableaux I et II ont donc été testées systématiquement, ainsi que leurs transformations (fonction puissance, forme polynomiale...), puis les combinaisons de ces variables présentant une signification écophysiologique a priori; l'analyse en régression multiple a permis de retenir les variables actives ou certaines de leurs transformations ou de leurs combinaisons lorsque celles-ci apportaient un gain de plus de $5 \%$ de la variance expliquée ( $r^{2}$ ajusté) par rapport à l'additivité simple. Dans les équations, les probabilités associées aux variables retenues sont toutes inférieures à 0,001 à une exception près $(P=$ 0,007 pour la $2^{e}$ variable de l'équation 1 ).

\section{RÉSULTATS}

\section{Clivages des données en plusieurs sous-ensembles climatiques (tableaux II et III)}

Un premier examen des données coupe par coupe fait ressortir la faible productivité de la pé- riode hivernale (novembre-février), particulièrement sous les bioclimats subhumide et humide, et la faible productivité mensuelle de mars à juillet sous bioclimat semi-aride. Pour permettre une analyse de finesse adaptée aux niveaux de productivité, il a été nécessaire de séparer résultats d'hiver et résultats de mars à juillet d'une part, résultats obtenus en semi-aride et résultats sous climats plus humides d'autre part. À défaut d'une telle précaution, les variables retenues traduiraient les variations des stations les plus productives; le modèle obtenu ne serait pas transposable aux stations plus faibles.

Par ailleurs, on a choisi de regrouper les résultats mensuels obtenus de mars à juillet dans les 3 bioclimats humides de façon à pouvoir tester les interactions entre variables climatiques mesurées à découvert; celles-ci sont le plus souvent très liées sur un même lieu; un découplage partiel est obtenu en considérant plusieurs lieux d'altitude et de climats différents.

Les 4 sous-ensembles suivants ont été retenus :

- récoltes de fin d'hiver en climats subhumide et humide, avec 30 mesures de productivité;

- récoltes de mars à juillet en climats subhumide et humide, avec 120 mesures;

- récoltes de fin d'hiver en climat semi-aride, avec 18 mesures;

- récoltes de mars à juillet en climat semi-aride, avec 63 mesures.

\section{Cas des parcours des ensembles subhumide et humide (chênaie verte pure, cédraie et pelouse asylvatique)}

\section{La production de matière sèche de la repousse d'hiver}

La productivité de la strate herbacée est très faible et dépend principalement de l'abondancedominance des légumineuses $(L g)$ en interaction avec le taux d'ouverture du couvert arboré : le produit $\left(L g^{0,5}\right)(100-R)$, où $R$ désigne le taux de recouvrement arboré, couvre $40 \%$ de la variance. La relation entre productivité et contribution des légumineuses est significativement mieux représentée par la fonction racine carrée que par une relation linéaire. Les contributions élevées des légumineuses sont associées aux recouvrements moyens, compris entre 20 et $60 \%$ (Qarro et de Montard, 1989); (points étoiles de la figure 1; cette figure sera expliquée plus loin). 
Tableau II. Synthèse des données climatiques en 1983 et 1984 . Ensemble (a) : bioclimats subhumide et humide : Indices climatiques de novembre-février. Ensemble (b) : bioclimats subhumide et humide : indices climatiques des 4 périodes de repousses (marsjuillet). Ensemble (c) : bioclimats semi-aride : chênaie verte à oxycèdre: indices climatiques de la période novembre-février. Ensemble (d) : climat semi-aride : indices climatiques des 4 périodes de repousses (coupes mensuelles de mars-juillet).

Année et mois $\quad J p \% \quad J r \% \quad$ ETR.ETP-1 $\theta p \quad R g$

\section{Ensemble a}

Chênaie verte pure

$\begin{array}{lrrrrr}1983 & 17 & 13 & 0,96 & 9,1 & 498 \\ 1984 & 21 & 15 & 1,00 & 9,5 & 498 \\ \text { Cédraie } & & & & & \\ 1983 & 17 & 17 & 0,86 & 6,4 & 498 \\ 1984 & 16 & 6 & 1,00 & 6,5 & 498 \\ & & & & & \\ \text { Pelouse } & & & & & \\ 1983 & 31 & 0 & 1,00 & 2,7 & 498 \\ 1984 & 17 & 2 & 0,87 & 4,3 & 498\end{array}$

\section{Ensemble b}

\begin{tabular}{|c|c|c|c|c|c|}
\hline \multicolumn{6}{|c|}{ Chênaie verte pure } \\
\hline \multicolumn{6}{|l|}{1983} \\
\hline mars & 25 & 30 & 1,00 & 12,0 & 720 \\
\hline avril & 8 & 48 & 0,92 & 10,6 & 857 \\
\hline mai & 17 & 58 & 0,92 & 16,3 & 947 \\
\hline juin juillet & 4 & 19 & 0,38 & 23,5 & 968 \\
\hline \multicolumn{6}{|l|}{1984} \\
\hline mars & 33 & 33 & 1,00 & 7,8 & 720 \\
\hline avril & 23 & 60 & 1,00 & 13,0 & 857 \\
\hline mai & 37 & 57 & 1,00 & 10,6 & 947 \\
\hline juin juillet & 2 & 18 & 0,65 & 24,6 & 968 \\
\hline
\end{tabular}

\section{Cédraie}

\begin{tabular}{lrrrrr}
1983 & & & & & \\
mars & 40 & 70 & 0,96 & 11,4 & 720 \\
avril & 20 & 22 & 1,00 & 9,0 & 857 \\
mai & 26 & 0 & 0,89 & 16,1 & 947 \\
juin juillet & 4 & 0 & 0,23 & 21,2 & 968 \\
& & & & & \\
1984 & & & & & \\
mars & 21 & 3 & 1,00 & 11,6 & 720 \\
avril & 26 & 60 & 1,00 & 13,2 & 857 \\
mai & 40 & 14 & 1,00 & 12,6 & 947 \\
juin juillet & 2 & 23 & 0,76 & 21,5 & 968 \\
\hline
\end{tabular}

\begin{tabular}{lrrrrr}
\hline Année et mois & $J p \%$ & $J r \%$ & ETR.ETP-1 $\theta p$ & $R g$ \\
\hline Ensemble b & & & & & \\
& & & & & \\
Pelouse asylvatique & & & & \\
1983 & & & & & \\
$\quad$ mars & 40 & 70 & 1,00 & 7,0 & 720 \\
$\quad$ avril & 22 & 22 & 1,00 & 8,7 & 857 \\
mai & 14 & 26 & 0,95 & 11,2 & 947 \\
juin juillet & 4 & 47 & 0,58 & 13,4 & 968 \\
& & & & & \\
1984 & & & & & \\
$\quad$ mars & 21 & 3 & 1,00 & 8,2 & 720 \\
$\quad$ avril & 26 & 13 & 1,00 & 12,2 & 857 \\
$\quad$ mai & 40 & 14 & 1,00 & 5,9 & 947 \\
juin juillet & 2 & 23 & 0,70 & 18,2 & 968 \\
& & & & &
\end{tabular}

\section{Ensemble c}

$\begin{array}{rrrrrr}1983 & 11 & 2 & 0,98 & 9,5 & 498 \\ 1984 & 17 & 9 & 0,97 & 10,3 & 498\end{array}$

$\begin{array}{lrrrrr}\begin{array}{l}\text { Ensemble d } \\ \text { 1983 }\end{array} & & & & & \\ \text { mars } & 30 & 10 & 0,97 & 15,8 & 720 \\ \text { avril } & 8 & 11 & 0,42 & 19,2 & 857 \\ \text { mai } & 9 & 0 & 0,18 & 24,3 & 947 \\ & & & & & \\ 1984 & & & & & \\ \text { mars } & 18 & 0 & 1,00 & 10,3 & 720 \\ \text { avril } & 16 & 0 & 0,71 & 17,8 & 857 \\ \text { mai } & 28 & 0 & 1,00 & 13,2 & 947 \\ \text { juin juillet } & 4 & 0 & 0,38 & 24,9 & 968 \\ \end{array}$

$J p \%$ : fréquence des jours de pluie $\% ; J r \%$ : fréquence des jours de rosée; ETR.ETP-1 : rapport de l'évapotranspiration du couvert (calculée) à l'évapotranspiration potentielle; $\theta p$ : température moyenne de la période (novembre à février, puis mensuelle) en ${ }^{\circ} \mathrm{C} ; R g$ : rayonnement global absolu en cal.cm${ }^{2} j^{-1}$. Périodes de repousse : période 1 , de novembre à février inclus; période 2, du 1er (1984) ou du 10 mars (1983) au 31 mars; période 3 , du $1^{\text {er }}$ avril au 3 mai; période 4 , du 4 mai au 7 juin; période 5 , du 8 juin au 20 juillet.

\section{Discussion}

L'effet favorable aux légumineuses d'un recouvrement arboré situé entre 20 et $60 \%$, est exprimé indirectement par leur abondancedominance. L'interaction avec le taux d'ouverture (100-R) indique une réponse positive de la productivité des légumineuses à contribution égale. La réponse au recouvrement est donc une combinaison complexe entre l'optimum de contribution des légumineuses et leur productivité croissant avec l'ouverture. 
Tableau III. Productivité de l'herbe (MS kg•ha-1.j-1) en fonction du bioclimat, du recouvrement arboré et de la période de repousse.

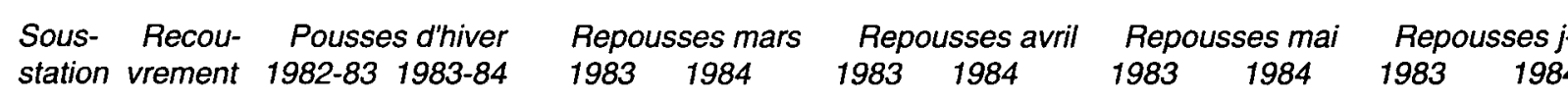

\begin{tabular}{|c|c|c|c|c|c|c|c|c|c|c|c|}
\hline \multicolumn{12}{|c|}{ Climat semi-aride (chênaie verte à oxycèdre) } \\
\hline 1 & 0 & 0,01 & 0,01 & 0,01 & 1,03 & 0,48 & 0,95 & 1,78 & 2,33 & 0 & 0,51 \\
\hline 4 & 0 & 0,17 & 0,06 & 1,58 & 1,46 & 2,00 & 2,30 & 3,71 & 5,07 & 0 & 1,93 \\
\hline 5 & 0 & 1,00 & 0,06 & 2,02 & 1,69 & 2,52 & 2,17 & 2,89 & 2,79 & 0 & 1,00 \\
\hline 9 & 0 & 0,57 & 0,24 & 6,07 & 4,05 & 6,02 & 4,98 & 2,72 & 12,86 & 0 & 1,50 \\
\hline 7 & 13 & 0,48 & 0,44 & 4,76 & 1,38 & 2,74 & 2,52 & 2,83 & 5,23 & 0 & 1,06 \\
\hline 3 & 19 & 0,17 & 0,21 & 1,23 & 1,21 & 0,61 & 1,40 & 1,08 & 2,01 & 0 & 2,62 \\
\hline 2 & 33 & 0,25 & 0,01 & 4,23 & 2,81 & 2,87 & 3,58 & 2,90 & 6,76 & 0 & 1,10 \\
\hline 6 & 62 & 0,81 & 0,24 & 2,78 & 1,64 & 3,63 & 2,22 & 2,15 & 6,04 & 0 & 1,54 \\
\hline 8 & 70 & 0,33 & 0,23 & 5,53 & 10,40 & 0,95 & 1,73 & 0,53 & 2,40 & 0 & 0,53 \\
\hline \multicolumn{12}{|c|}{ Climat subhumide (chênaie verte pure) } \\
\hline 12 & 0 & 0,95 & 1,50 & 5,45 & 3,73 & 5,74 & 11,17 & 6,78 & 9,85 & 2,74 & 2,66 \\
\hline 13 & 0 & 0,60 & 0,72 & 5,71 & 3,69 & 7,82 & 6,20 & 6,00 & 11,07 & 2,44 & 2,64 \\
\hline 14 & 19 & 0,70 & 1,69 & 9,31 & 4,70 & 3,95 & 6,07 & 6,49 & 4,90 & 3,13 & 2,45 \\
\hline 16 & 20 & 0,52 & 0,73 & 5,93 & 3,73 & 3,22 & 3,55 & 3,55 & 5,76 & 1,06 & 1,31 \\
\hline 11 & 38 & 1,35 & 0,68 & 8,30 & 2,00 & 7,53 & 2,69 & 2,83 & 10,16 & 1,21 & 1,08 \\
\hline 17 & 39 & 0,88 & 1,11 & 8,11 & 1,31 & 5,17 & 2,97 & 8,10 & 6,10 & 1,55 & 1,76 \\
\hline 10 & 62 & 0,41 & 0,85 & 8,81 & 2,88 & 7,41 & 5,22 & 4,47 & 7,31 & 1,70 & 1,96 \\
\hline 15 & 91 & 0,17 & 0,04 & 0,58 & 1,54 & 0,65 & 0,40 & 0,36 & 0,37 & 0,24 & 0,30 \\
\hline \multicolumn{12}{|c|}{ Climat humide frais (cédraie) } \\
\hline 21 & 0 & 0,39 & 0,37 & 2,90 & 1,59 & 2,60 & 3,33 & 2,08 & 3,07 & 1,46 & 1,24 \\
\hline 23 & 0 & 0,68 & 0,64 & 2,38 & 2,28 & 3,28 & 4,55 & 1,60 & 4,43 & 2,04 & 1,67 \\
\hline 20 & 65 & 0,20 & 0,41 & 1,41 & 0,74 & 3,88 & 2,74 & 2,76 & 1,53 & 0,47 & 0,86 \\
\hline 18 & 70 & 0,55 & 0,18 & 3,97 & 0,57 & 1,98 & 2,27 & 3,68 & 2,25 & 0,62 & 1,58 \\
\hline 19 & 75 & 1,27 & 0,32 & 3,98 & 1,66 & 3,03 & 8,03 & 5,18 & 2,96 & 0,93 & 1,47 \\
\hline 22 & 83 & 0,26 & 0,22 & 1,19 & 0,54 & 0,77 & 0,59 & 1,08 & 0,63 & 0,77 & 0,78 \\
\hline \multicolumn{12}{|c|}{ Climat froid (pelouse) } \\
\hline 24 & 0 & 0,90 & 0,67 & 4,84 & 2,76 & 12,00 & 4,40 & 8,09 & 7,13 & 2,91 & 4,05 \\
\hline
\end{tabular}

Autres variables. La reprise des pluies en automne (tableau IV), les possibilités de pénétration et de stockage dans le sol constituent un deuxième ensemble interactif : le produit « $P_{s o} S g$ $\operatorname{Pr}^{2}$, où $P_{\text {so }}$ désigne la somme des précipitations de septembre et octobre, $S g$ le taux de sable grossier de l'horizon supérieur, et Prla profondeur du sol, couvre $14 \%$ de la variance.

Le nombre limité de résultats d'hiver, 30 pour 5 variables élémentaires, interdit l'introduction d'autres variables. L'équation de régression finale est la suivante :

$$
\begin{aligned}
\text { MS par jour } & =1,7.10^{-3}\left(\operatorname{Lg}^{0,5}\right)(100-R) \\
& +4,8.10^{-8} P_{\text {so }} S g P r^{2} \\
r^{2} \text { ajusté }= & 0,549
\end{aligned}
$$

\section{La production de matière sèche par coupe de mars à juillet}

L'analyse des résultats des 4 coupes échelonnées de mars à juillet a permis d'étudier l'effet des importantes variations de température moyenne, d'énergie globale absolue journalière moyenne, de niveau d'alimentation en eau $\left(E T R E T P^{-1}\right)$ et de fréquence des jours de pluie et de rosée de chacun des 4 intervalles entre coupes étudiés en 1983 et 1984, soit mars, avril, 1 er mai-5 juin et 5-juin-20 juillet.

La production obtenue apparaît comme la résultante additive de 3 effets principaux : l'interaction de l'énergie incidente et du recouvrement arboré; l'interaction «niveau d'alimentation en eau $x$ état de la matière organique»; l'interaction 


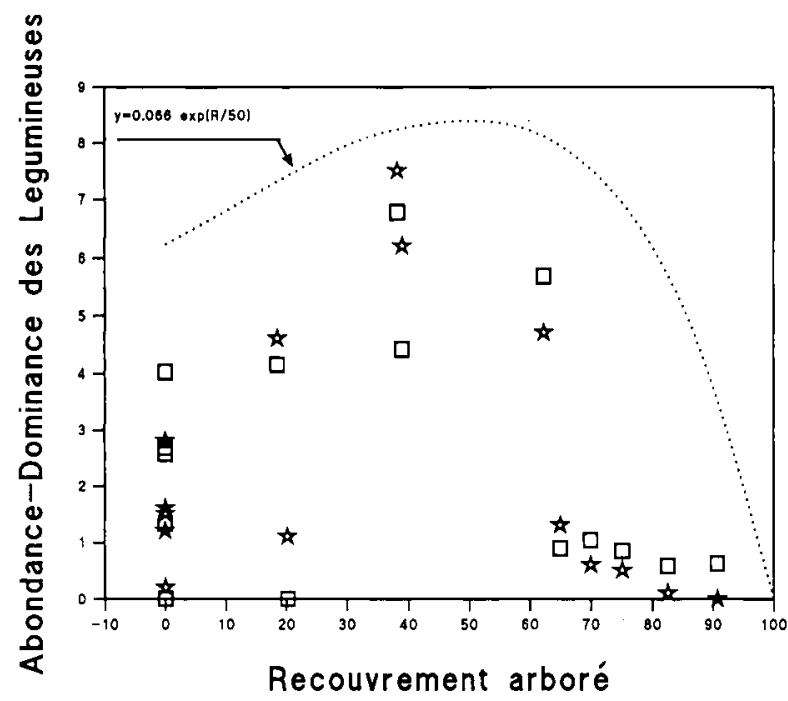

Fig 1. Somme des abondances-dominances des légumineuses dans le subhumide et l'humide : valeur observée (étoile) et valeur calculée (carré) en fonction des interactions "Recouvrement $\times \mathrm{pH} \times \mathrm{K}$ échangeable»: $\mathrm{Lg}=-2,9+0,030$ $K e(\rho H-5,6)(100-R) e^{R / 50}$; avec $r^{2}$ ajustè $=0,799$ pour les 15 sous-stations.

Tableau IV. Somme des pluies de septembre et octobre $(\mathrm{mm})$ :

\begin{tabular}{lrc}
\hline Station bioclimatique & 1982 & 1983 \\
\hline & & \\
Chênaie verte à oxycèdre & 79 & 18 \\
Chênaie verte pure & 125 & 40 \\
Cédraie & 161 & 68 \\
Pelouse asylvatique & 139 & 69
\end{tabular}

«niveau d'alimentation en eau $x$ contribution des légumineuses $x \mathrm{~K}$ échangeable".

L'ensemble de ces 3 variables couvre $52,3 \%$ de la variance totale pour 120 observations. L'équation de régression est la suivante (la succession des variables dans l'équation se lit verticalement) :

$$
\begin{aligned}
& M S= \\
& t \\
& -2,19 \\
& +41,5 \text { (ETR.ETP-1) N.C-1 } \\
& +0,28\left(E T R . E T P^{-1}\right) L g^{0,5} . K e \\
& 5,02 \\
& +1,110^{-3}(R g-679) \theta^{-1}(100-R) \mathrm{e}^{R / 90} \\
& \left(r^{2} \text { ajusté }=0,523\right)
\end{aligned}
$$

Dans cette équation, MS est la production de matière sèche par coupe exprimée en $\mathrm{kg}^{\circ} \mathrm{ha}^{-1} \bullet \mathrm{j}^{-1}$; elle est exprimée ainsi pour que les résultats issus des périodes intercoupes de durées différentes puissent être comparés directement; ETR.ETP-1 est le niveau d'alimentation en eau calculé sur la période pour une réserve du sol de $100 \mathrm{~mm}, N . \mathrm{C}^{-1}$ est l'inverse du rapport [carbone]/[azote] de l'horizon supérieur, $L g$ l'abondance-dominance des légumineuses (chaque espèce étant notée de 0 à 5), Ke le taux de potassium échangeable de l'horizon supérieur du sol, $R g$ le

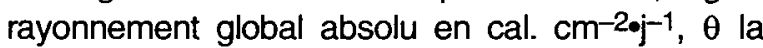
température moyenne de la période intercoupe et $R$ le recouvrement arboré en (\%). Le quotient du nombre d'observations au nombre de variables est de 40 pour les variables élaborées, et de 17 pour les variables élémentaires.

\section{Discussion}

En première analyse, le taux de variance expliquée paraît modeste; cependant, il est très conséquent eu égard à la grande hétérogénéité des sols concernés et à la difficulté des mesures qui en résulte. Les variables testées concernent des phénomènes connus; l'intérêt de cette régression multiple est de confirmer leur pertinence dans un contexte où elles entrent en interaction et de pouvoir identifier les interactions les plus puissantes. II s'agit ici d'interactions vraies, la forme additive ayant été largement supplantée par la forme produit dans les tests. Du point de vue statistique, la hiérarchie entre les 3 variables est peu marquée (valeurs de $t$ voisines).

Du point de vue agronomique, l'interprétation est relativement simple : les 2 premières variables concernent l'interaction «eau x azote» en distinguant 2 sources :

- la minéralisation de la matière organique est reflétée par le rapport $[\mathrm{N}] /[\mathrm{C}]$ de l'horizon supérieur; elle est moins active pour les $C . N^{-1}$ élevés, d'autant moins que les niveaux d'alimentation en eau sont faibles;

- la fixation symbiotique est représentée par la deuxième variable; elle apparaît ici limitée par le niveau d'alimentation en eau, et ce d'autant plus que les disponibilités en $K$ sont faibles, en accord avec Shansum-Noor et al (1989). Ainsi que cela a été souligné pour la pousse d'hiver, la variable $L g$ indique un effet du recouvrement arboré; plus précisemment, l'abondance-dominance des légumineuses est soumise à l'interaction du taux de $\mathrm{K}$ échangeable de l'horizon supérieur du sol avec son $\mathrm{pH}$ et le taux de recouvrement arboré; l'équation de régression est la suivante : $L g=$ 
$-2,9+0,030 \mathrm{Ke}(\mathrm{pH}-5,6)(100-R) e^{R / 50}$; avec $r^{2}$ ajusté $=0,799$ pour les 15 sous-stations : le quotient est de 5 observations par variable élémentaire. Cette relation met en évidence un maximum de légumineuses pour un recouvrement de $50 \%$ (fig 1) variable en fonction croissante de l'interaction «nutrition potassique $\times \mathrm{pH}$ ». Les espèces présentes en abondance dans le sousbois sont Anthyllis vulneraria, Trifolium campestre et Medicago suffruticosa (Qarro, 1985); il faut souligner que cette aptitude de certaines légumineuses à s'adapter à l'ombrage est mise à profit en Nouvelle Zélande dans la culture mixte Pinus radiata + Lotus pedunculatus pratiquée sous des climats doux et humides (West et al, 1988).

La troisième variable concerne la ressource énergétique, et sa modulation par le recouvrement arboré; par ailleurs, elle est valorisée en fonction inverse de la température, les températures excessives étant un facteur de forte augmentation du déficit de saturation de l'air, de fermeture des stomates et de ralentissement des échanges gazeux et de la photosynthèse (Murata et lyama, 1963; Woledge et Parsons, 1986). II a été souligné précédemment que les déficits hydriques correspondaient, entre mars et juillet, à des températures supérieures à $17^{\circ} \mathrm{C}$ (Qarro et de Montard, 1989).

La résultante des effets du recouvrement arboré est le mieux exprimée par la fonction $(100-R)$ $e^{R / 90}$; la courbe représentative de cette fonction appartient à une famille de courbes $Y=(100-R)$ $e^{R / B}$ (fig 2) qui permet d'exprimer un effet dissymétrique du recouvrement; le meilleur ajustement est obtenu pour $B=90$; à cette valeur correspond un léger maximum pour $R=10$, et une quasi-absence d'effet du recouvrement entre 0 et $40 \%$; avec 60 et $80 \%$ de recouvrement, la productivité est encore à 80 et $40 \%$ de celle du découvert, respectivement.

Ainsi, l'utilisation de l'énergie incidente est conditionnée par le taux de recouvrement d'une manière complexe : le couvert arboré, non seulement diminue la quantité d'énergie incidente et intercepte une partie des pluies (Aussenac et Boulangeat, 1980), mais encore compense partiellement ces pertes d'énergie et d'eau par une meilleure efficience (Ovalle et Avendano, 1987); il constitue en effet une protection contre l'insolation directe, tamponne les pointes diurnes d'évapotranspiration et la fermeture des stomates au niveau du couvert herbacé sous-jacent, retarde le dessèchement du sol.

Berbigier et al (1991) ont montré qu'au fur et à mesure de l'aggravation du stress hydrique au cours de l'été, l'évaporation de la molinie devient plus importante que celle du pin maritime qui la couvre, bien que le déficit de saturation de l'air en sous-bois soit à peine moins marqué qu'audessus des houppiers; la molinie utilise mieux les pluies de fin d'été que le pin, ce qui suggère une meilleure compétitivité pour l'absorption de l'eau arrivant dans l'horizon supérieur : une telle relation "pin-molinie" au niveau racinaire ne peut être généralisée, mais est une hypothèse plausible pour les relations "cèdre-herbe" ou "chêne vert-herbe" qu'il faudrait tester compte tenu de la réponse au recouvrement observée en Moyen Atlas.

Le couvert constitue aussi un écran au rayonnement nocturne en direction du ciel noir et limite ainsi les gelées au sol. À ces phénomènes, s'ajoute le rôle de l'arbre dans la maintenance de propriétés du sol plus favorables, mis en évidence par Joffre (1987).

Une quatrième variable, d'effet positif, le rapport de la fréquence des jours de rosée au recouvrement, $J r \%(R+1)^{-1}$, aurait pu être introduite très significativement dans l'équation bien qu'elle ne permette pas d'accroître le taux de variance expliquée de plus de $3 \%\left(r^{2}=0,548\right)$; il est intéressant de la signaler pour orienter de futures investigations plus fines sur le rôle de l'arbre. En milieu sec, la rosée peut jouer un rôle dans l'alimentation en eau qu'il faudrait préciser en relation avec l'effet d'écran de la couronne des arbres et l'aptitude du sol sec à capter l'humidité atmosphérique ( $M$ Étienne com pers).

\section{Cas des parcours du semi-aride (chênaie verte à oxycèdre)}

\section{Production d'hiver en semi-aride}

Les quantités de matière sèche accumulées en hiver et récoltées en début mars n'ont pas de relation apparente avec le taux de recouvrement arboré, bien que celui-ci varie de 0 à $70 \%$. La différence entre les 2 années correspond à l'importance des précipitations tombées en septembre et octobre avant la période des jours courts et des faibles températures (tableau IV). Ces pluies ont été d'autant mieux valorisées que le sol était riche en matière organique : la relation " $M S / \mathrm{j}=1,71.10^{-3} P_{S O}(R)$ " où MS est la matière sèche récoltée $\left(\mathrm{kg}^{\mathrm{s}} \mathrm{ha} \mathrm{a}^{-1} \mathrm{j}^{-1}\right), P_{\text {so }}$ la somme des précipitations cumulées de septembre et octobre et $\mathrm{N}$ la teneur en azote de l'horizon supérieur du sol-, couvre $54,7 \%$ de la variance avec 18 observations ( $r^{2}$ ajusté $=0,547$ ); le ratio est de 9 obs- 
ervations par variable élémentaire; la faible teneur en azote apparaît comme facteur limitant de la valorisation des pluies.

\section{La production de matière sèche par coupe de mars à juillet}

Durant cette période la production de MS par coupe et par jour apparaît comme la résultante d'une interaction entre l'énergie incidente, la fréquence des jours de pluie et le recouvrement arboré; la fonction la plus représentative des effets du couvert est (100-R) $e^{R / 50}$; elle appartient à la même famille de courbes que celle observée sous climats subhumide et humide, mais le maximum est situé vers $50 \%$ de recouvrement au lieu de 10 (fig 2). Par ailleurs, l'une des sousstations située à découvert (sous-station 9), extériorise une production significativement plus importante que n'indique la première variable. On a donc intégré un terme correctif pour celle-ci dans l'équation pour en tenir compte :

$\mathrm{MS} / \mathrm{j}=$

$0,81+97.10^{-6} \mathrm{Jp} \% R g(100-R) e^{R / 50}+\left[91,4 \cdot 10^{-4}\right.$ $J p \%(R g-600)]_{s 9}$

$r^{2}$ ajusté $=0,636$, avec 62 observations après exclusion d'une valeur discordante. Le quotient est de 31 observations par variable élaborée et de 15 observations par variable élémentaire. Dans cette équation, MS désigne la production de matière sèche en $\mathrm{kg}^{\circ} \mathrm{ha}^{-1} \cdot \mathrm{j}^{-1}, J \mathrm{p} \%$ la fréquence des jours de pluie des périodes entre 2 coupes en $\%, R g$ le rayonnement global absolu, $R$, le taux de recouvrement arboré. Le terme correctif afférent à la sous-station (9) est expliqué par sa position dans un petit vallonnement qui favorise le passage des eaux de ruissellement.

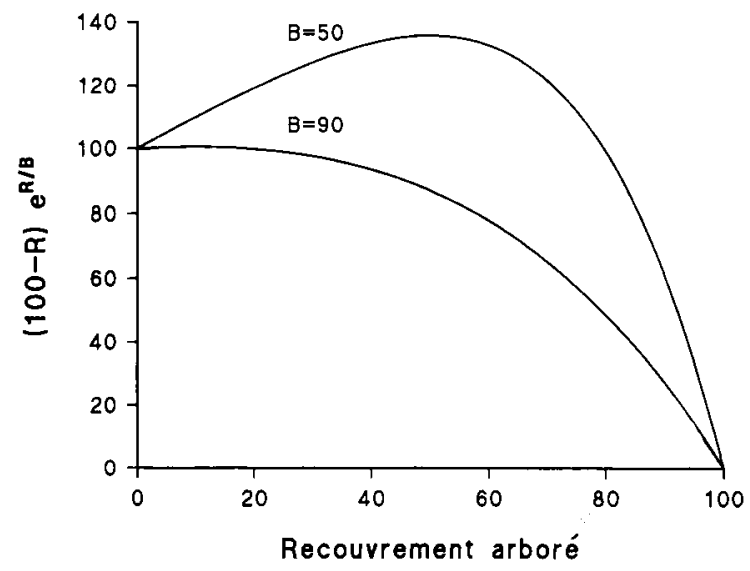

Fig 2. (a) Courbes représentatives de la fonction $Y=(100-$ $X) \mathrm{e}^{\mathrm{X} / \mathrm{B}}$ pour $B=50$ et $B=90$.
Le taux de variance expliquée est satisfaisant eu égard à l'hétérogénéité du sol et à la faiblesse des quantités récoltées, qui affectent les mesures d'une forte erreur relative.

Sous ce climat semi-aride, et pour les 7 périodes mensuelles observées, la proportion de jours de pluie est, de beaucoup $(+12 \%)$, plus explicative de la $M S$ récoltée que le niveau d'alimentation hydrique ETR.ETP-1.

Avec un maximum de production pour $50 \%$ de recouvrement, l'effet de protection par le couvert arboré apparaît plus marqué qu'en climat humide. Le constat d'un effet bénéfique d'un couvert important dans ce type de climat rejoint les conclusions de Frost et Mc Dougald (1989) sous couvert de Quercus sp en Californie et d'Ovalle et Avendano (1987) sous Acacia caven.

\section{Convergences et divergences observées entre bioclimats}

Des variantes importantes dans la nature et la hiérarchie des variables et le niveau de leurs interactions, en relation avec la saison et le bioclimat considérés ont ainsi été mises en évidence; une comparaison des résultats obtenus respectivement en climat semi-aride et en climats subhumide et humide est maintenant possible.

\section{La production de matière sèche en hiver}

Elle dépend du cumul des pluies de septembre et octobre; en interaction positive avec le taux de sable grossier et la profondeur sous climats subhumide et humide où les sols sont lourds; en interaction positive avec le taux d'azote dans les sous-stations du bioclimat semi-aride où les sols sont pauvres et beaucoup plus légers.

Dans le subhumide et l'humide, elle dépend aussi d'interactions positives «légumineuses $x$ éclairement».

\section{La production de matière sèche de mars à juillet}

Interaction "énergie globale incidente $x$ recouvrement arboré»

La ressource énergétique explique une large part des variations observées dans les 2 ensembles climatiques, bien que, a contrario, on observe aussi un effet favorable du recouvrement arboré (fonctions $e^{R / 90}$ et $e^{R / 50}$ ); cet effet est plus fort en climat semi-aride pour le compartiment non fixateur : l'exposant y est égal à $R / 50$ au lieu de $R / 90$ dans le subhumide/humide; toutefois, dans 
ce dernier, l'exposant $R / 50$ apparaît aussi, dans l'expression de l'abondance-dominance des légumineuses; il y a donc 2 compartiments, l'un non fixateur, l'autre fixateur d'azote, extériorisant des réponses différentes au couvert arboré.

L'humidité du mésoclimat - caractérisée au niveau de postes météorologiques situés à découvert - s'exprime différemment dans les 2 ensembles :

- en climats subhumide et humide, le niveau d'alimentation en eau, assez fortement lié à la fréquence des pluies ( $r=0,713)$, est dominant; il interagit avec l'état de la matière organique d'une part, avec l'abondance-dominance des légumineuses et des disponibilités en $\mathrm{K}$ d'autre part; cette dernière interaction correspond bien aux facteurs connus de la fixation d'azote;

- en climat semi-aride, l'effet de la fréquence des pluies est dominant et masque celui du niveau d'alimentation en eau; il n'y a pas d'action significative des légumineuses.

\section{Relation à la température}

Sous les climats subhumide et humide, la valorisation de l'énergie incidente sous forme d'accumulation de MS est contrariée par les températures trop élevées. Cet effet est expliqué en rapprochant les courbes de réponse à la température obtenues par divers auteurs (Murata et lyama, 1963; Woledge et Parsons, 1986; cités par Lafarge, 1991) : le déficit de saturation de l'air tend à croître spontanément avec l'élévation de la température, même en l'absence de déficit hydrique dans le sol, provoquant un ralentissement des échanges gazeux et de la photosynthèse. En effet, les jours sans pluie, la teneur en vapeur d'eau est largement déterminée par la température minimale de la nuit précédente.

En climat semi-aride où les températures élevées correspondent à des déficits hydriques très marqués, déficit de saturation de l'air et déficit hydrique ne sont pas séparables; de plus, les pluies sont très irrégulières : leur fréquence domine le modèle.

\section{Nature et place des caractéristiques du sol dans l'équation}

En climats subhumide et humide, la teneur en $\mathrm{K}$ et le rapport $[C] .\left[N^{-1}\right]$ entrent significativement dans le jeu des interactions avec le niveau d'alimentation en eau.

En climat semi-aride, la sous-station 9 , mieux alimentée en eau de par sa position topographique, est la seule référence aux conditions microlocales autres que le recouvrement arboré.
Dans les 2 cas, la grande hétérogénéité des sols n'a permis qu'une intégration incomplète de leurs caractéristiques dans le fonctionnement de la végétation herbacée; cela explique en grande partie les valeurs modestes de $r^{2}$ ajusté; elles sont cependant suffisamment élevées pour en souligner d'une manière hautement significative les principales.

\section{Rôle des légumineuses}

L'interaction positive entre contribution des légumineuses, $K$ échangeable et niveau d'alimentation en eau est forte dans le subhumide et l'humide qui sont nettement plus riches en $\mathrm{K}$ et mieux alimentés en eau que le semi-aride. Dans ce dernier, la précocité et l'importance des déficits hydriques, la pauvreté des sols en potassium ne permettent pas l'expression d'une activité particulière aux légumineuses en l'état actuel des parcours.

\section{CONCLUSIONS}

\section{Limites de validité des résultats}

L'intérêt des observations d'hiver reste limité par le petit nombre de données, sur 2 hivers seulement :

- le modèle est provisoire;

- le modèle concernant les productions de printemps et début d'été couvre 8 périodes intercoupes ( 7 dans le semi-aride) réparties sur les 2 campagnes 1983 et 1984; il repose donc sur une base beaucoup plus étayée mais qui nécessite encore une validation dans des milieux et pour des années climatiques, analogues ou intermédiaires, c'est-à-dire sur un gradient d'altitude de 800 à $2200 \mathrm{~m}$ d'altitude et pour des climats où la température moyenne mensuelle n'excède pas $27^{\circ} \mathrm{C}$ de mars à juillet. Les convergences constatées entre les 2 climats constituent une première validation; leur divergences soulignent les limites de la généralisation.

La stabilité des résultats a été étudiée en constituant 2 groupes dans chacun des 4 sousensembles $a, b, c$ et $d$ (prélèvement randomisé d'un résultat sur 2 dans les données de chaque mois). Les variables explicatives sont conservées à l'exception du produit $P_{s o} S g P^{2}$ pour l'1 des 2 groupes concernés (hiver, bioclimats humide et subhumide); les index de détermination ( $r^{2}$ ajusté) sont voisins de ceux des 4 équations principales. 
Des investigations nouvelles seraient utiles pour les climats plus arides afin de définir l'optimum de recouvrement jusqu'à la limite de l'aire boisée, notamment pour l'arganier.

\section{Intérêt de la modélisation}

Les premières conclusions (Qarro et de Montard, 1989) se trouvent confirmées et affinées par la modélisation sur les données mensuelles qui a permis d'exprimer des interactions complexes entre recouvrement arboré, sol et climat : la forme des courbes de réponse au taux de recouvrement est mise en évidence en rapport avec l'aridité du climat. Dans le contexte du Moyen Atlas où un aménagement sylvo-pastoral permettant d'améliorer la ressource pastorale en respectant la production ligneuse a été défini comme prioritaire par les gestionnaires, une nouvelle gestion sylvicole tenant compte du potentiel herbacé en fonction du recouvrement arboré peut être mise au point sur cette base scientifique.

Toutefois, il reste à approfondir les réponses en fonction des essences forestières, chêne vert ou cèdre, et la contribution pastorale des rejets de chêne vert après dépressage du taillis.

\section{L'optimisation des 2 composantes de la production}

La modélisation a permis de confirmer que la production mesurée sous les bioclimats subhumide et humide est issue de 2 compartiments

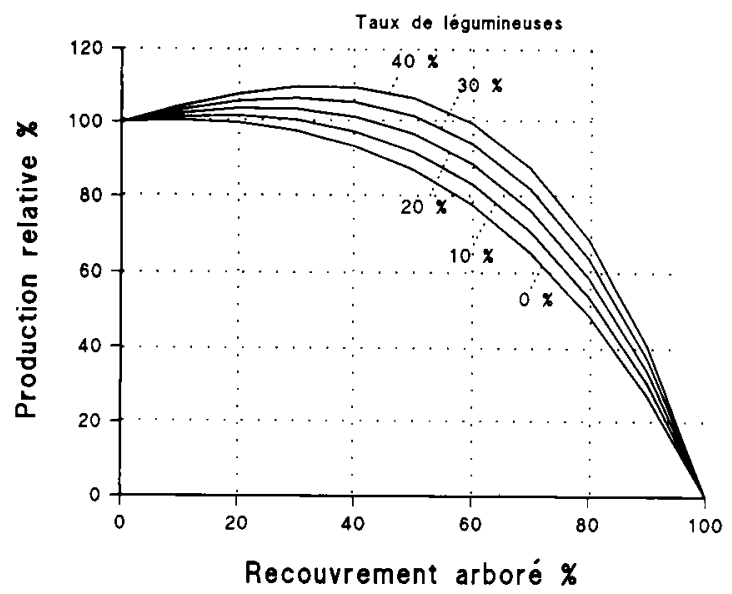

Fig 3. Abaque de la production relative de la strate herbacée en relation avec le taux de recouvrement arboré pour différentes hypothèses de contribution relative pondérale des légumineuses. prairiaux qui fonctionnent différemment : l'un constitué par des espèces fixatrices d'azote dont la contribution est maximale pour un recouvrement de $50 \%$; l'autre par des espèces non fixatrices pour lesquelles le maximum de production est obtenu vers $10 \%$ de recouvrement.

La résultante de ces comportements différents peut être simulée sur une abaque dont les paramètres dépendent de la contribution relative des 2 groupes d'espèces (fig 3 ). Dans la mesure où la proportion de légumineuses pourrait être accrue et maîtrisée (rythme de la pâture, fertilisation, sursemis), l'abaque montre un accroissement du taux optimal de recouvrement arboré quand le taux de recouvrement des légumineuses s'accroît. La validation nécessite maintenant une phase expérimentale mettant en jeu une fertilisation modeste et répétée sur 4-5 ans, en l'élément le plus limitant pour la fixation symbiotique $-K$ dans le cas présent ( $P$ dans d'autres cas : Osman et al 1991) - notamment dans les zones sylvo-pastorales les plus fréquentées.

Cependant, en l'état actuel de ces parcours, le niveau de productivité est très bas eu égard aux précipitations : la question du niveau réel de la nutrition en éléments majeurs doit être examinée pour orienter l'expérimentation; une prochaine recherche analysera les disponibilités en azote et leur rôle limitant pour la production herbacée.

\section{RÉFÉRENCES}

Aussenac G, Boulangeat C (1980) Interception des précipitations et évapotranspiration réelle dans des peuplements de feuillus et de résineux. Ann Sci For $37,91-108$

Berbigier P, Diawara A, Loustau D (1991) Étude microclimatique de l'effet de la sécheresse sur l'évaporation d'une plantation de pins maritimes et du sous-bois. Ann Sci For 2, 157-177

Clary WP (1975) Range management and its ecological basis in the Ponderosa pine type Arizona: the status of our knowledge. Fort Collins, USDA For Serv (Res pap) IM 158, 35 p

Dodd CJ, Mc Lean A, Brinck VC (1972) Grazing values as related to tree crown covers. Can J For Res 2, 185-189

Eason WR (1991) Effect of tree leaf litter on sward botanical composition and growth. In: Agroforestry: Principles and practice (Janvis PG, ed) Elsevier, Amsterdam, 165-172

Engle DM; Stritzke JF, Claypool PL (1987) Herbage standing crop around eastern redcedar trees. J Range Manage 40, 237-239 
Étienne M, Msika B (1987) Rôle écologique de l'arbre dans un taillis de Quercus pubescens soumis à différentes intensités d'éclaircie. In: Rapport sur dehesas et systèmes sylvo-pastoraux. INRA Ecodev Avignon, 9-20

Frost EW, Mc Dougald NK (1989) Tree canopy effects on herbaceous production of annual rangeland during drought. J Range Manage 42, 281-283

Gaines EM, Campbell RS, Brasington JJ (1954) Forage production on longleaf pine stands of southern Alabama. Ecology 35, 59-62

Hubert D (1989) Recherches sur la gestion écologique des ressources sylvopastorales du système pluristratifié à Quercus pubescens et herbacées du Causse Méjean. Rapp final rech, CEPE Montpellier, $58 \mathrm{p}$

Joffre R (1987) Contraintes du milieu et réponses de la végétation herbacée dans les dehesas de la Sierra Norte. Thesis, Montpellier, $201 \mathrm{p}$

Knowles RL (1989) New Zealand experience with silvo-pastoral systems: a review. In: Agroforestry: principles and practice (Jarvis PG, ed) Elsevier, Amsterdam, 251-268

Lafarge M (1991) Morphogénèse et croissance d'une orge de printemps cultivée à différences altitudes. Notion de dynamique du peuplement. Thèse (à paraître)

Leithead (1970) A guide to forage resources of grazable woodlands in the southern coastal plains. $\mathrm{In}$ : Tree growth and forest soils. Proc 3rd north Am forest soil conf, North Carolina State Univ, Raleigh NC, 1968, 449-459

Lewis CE (1989) Herbage yield response to the maturation of a slash pine plantation. $J$ Range Manage 42, 191-195

Murata $Y$, lyama $J(1963)$ Studies on the photosynthesis of forage crops II. Influence of air temperature upon the photosynthesis of some forage and grain crops. Proc Crop Sci Soc Jpn 31, 315-322

Osman AE, Cocks PS, Russi L, Pagnotta MA (1991) Response of Mediterranean grassland to phosphate and stocking rates: biomass production and botanical composition. J Agric Sci Camb 116, 37-47

Ovalle C, Avendano J (1987) Interactions entre la strate ligneuse et la strate herbacée dans les formations d'Acacia-Caven. I. Influence de l'arbre sur la composition botanique, la production et la phénolo- gie de la strate herbacée. Acta Oecol/Oecol Plant $8,385-404$

Ovalle C, Avendano J (1988) Interactions de la strae herbacée et de la strate ligneuse dans les formations d'Acacia-Caven. II. Influence de l'arbre sur quelques éléments du milieu : microclimat et sol. Acta Oecol/Oecol Plant 23, 113-134

Qarro M (1985) Étude de la productivité du tapis herbacé des parcours de la zone d'Aîn-Leuh au Maroc (Moyen Atlas-plateau Central). Thèse en écologie, Fac St Jérôme, Univ Aix-Marseille, $258 p$

Qarro M, Montard FX de (1989) Étude de la productivité des parcours de la zone d'Aïn-Leuh (Moyen Atlas, plateau Central). I. Effets de la fréquence d'exploitation et du taux de couvert arboré sur la productivité herbacée. Agronomie 9, 477-487

Richard P (1987) Effet du pâturage sur la végétation du sous-bois dans les Landes de Gascogne. Thèse écol, Univ Rennes, $157 \mathrm{p}$

Shamsum-Noor L, Robin C, Shontz D, Heulin T, Guckert A (1989) Effet d'un déficit hydrique sur le trèfle blanc. II. Rôle d'un apport de potassium. Agronomie 9, 251-257

Vacher $J$, Joffre R, Ortega F, Fernandez Ales R, Martin Vicente A (1985) L'organisation de r'espace dans la Sierra Norte de Séville (Sierra Morena) et les problèmes actuels des dehesas. Rev Géogr Pyr Sud-Ouest 56, 179-201

West G, Percival N, Dean M (1988) Oversowing legumes and grasses for forest grazing. In: Proc Agrofor symp Rotorua. N Z FRI Bull 139, 220 p

Woledge J, Parsons AJ (1986) The effect of temperature on the photosynthesis of ryegrass canopies. Ann Bot 57, 487-497

Woods RF, Betters DR, Mogren EW (1982) Understory herbage production as a function of Rocky Mountain aspen stand density. J Range Manage $35,380-381$

Zimka JR, Stachurski A (1975) Regulation of C and N transfer to the soil of forest ecosystems and the rate of litter decomposition. Bull Acad Pol Sci, Serr Sci Biol II 24, 127-132

Zimka JR, Stachurski A (1976) Vegetation as a modifier of carbon and nitrogen transfer to soil in various types of forest ecosystems. Ecol Pol 24, 493514 